\title{
FDA approves first single-dose antimalarial
}

On July 20, the US Food and Drug Administration approved GlaxoSmithKline's Krintafel (tafenoquine), a single-dose treatment to prevent relapses of Plasmodium vivax, a malaria parasite that causes more than eight million infections annually. "This drug could be transformative," says David Reddy, executive director of Medicines for Malaria Venture, GlaxoSmithKline's partner in the development of Krintafel. On August 9, the FDA also gave the go-ahead to 60 Degrees Pharmaceuticals for a six-month dosing regimen of tafenoquine tablets, commercialized as Arakoda, to prevent malaria in adults, including travelers and residents of disease-endemic countries.

Krintafel is the first new treatment for $P$. vivax malaria in over 60 years. The drug is a long-acting synthetic analogue of the malaria drug primaquine. It is a new chemical entity in the aminoquinoline class, produced by adding methoxy groups at two positions and a 3-trifluoromethylphenoxy substitution at another position of primaquine's quinoline ring. These modifications allow it to persist longer in the bloodstream than primaquine, which translates into a pharmacodynamic benefit. One dose of Krintafel replaces the current standard of care for relapsing infections, a 14-day course, which patients find difficult in terms of compliance, says Reddy.

Both tafenoquine and primaquine are active against hypnozoites, dormant cells of the malaria parasite that reside in the liver. When reactivated, hypnozoites cause both relapse and renewed disease transmission. After a bite from an infected mosquito, Plasmodium species multiply first in the liver and then inside red blood cells. Within red blood cells, the parasites produce toxic substances that are ultimately released into the blood, triggering fever and other malaria symptoms.

The precise mechanism of action of these drugs is not known: a leading hypothesis is that Krintafel may exert its effect by inhibiting hematin polymerization and inducing apoptosis-like death of the Plasmodium parasite. "There are a number of hypotheses, and that's a leading one," Reddy says. "We can say the mechanism would be the same as primaquine because the two drugs are very closely related chemically."

An alternative hypothesis is that tafenoquine is activated in the red blood cell itself to target the parasite through oxidative stress, adds Stephen Toovey, head of the virology- and tropical-disease-focused consultancy Pegasus Research.

Arakoda from 60 Degrees is the first preventive drug approved in almost 20 years. It kills the parasites in both blood and liver, and because it protects against both major types of the malaria parasite, $P$. vivax and P. falciparum, doctors can prescribe it to protect travelers visiting most zones in which malaria is endemic. Arakoda was originally discovered by researchers at the Walter Reed Army Institute of Research and codeveloped with 60 Degrees to bring the drug to the US market as a once-aweek oral dosing regimen. Toovey says that for prophylaxis in travelers, "tafenoquine broadens the choices open to physicians and patients."

The limitation for both Krintafel and Arakoda, however, is that they are contraindicated in glucose-6-phosphate dehydrogenase (G6PD)-deficient individuals, as the enzyme deficiency increases their risk of hemolytic anemia. G6DP is an inherited disorder affecting more than 400 million people worldwide. It is especially prevalent in areas with endemic malaria because the defect appears to offer some protection against malaria infection.

Mark Ratner

\section{Around the world in a month}

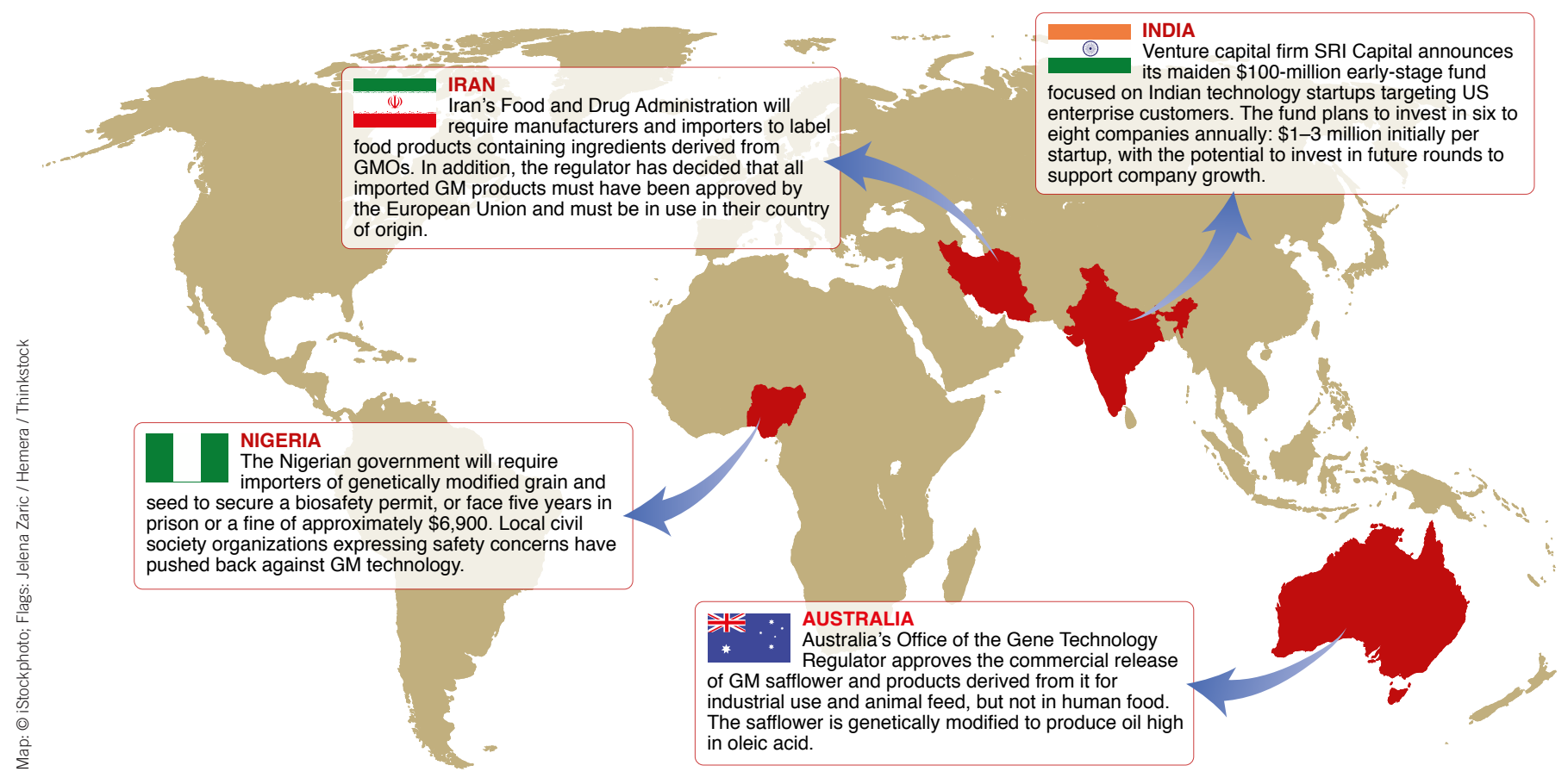

